\title{
Semiclassical approach to the ac conductance of chaotic cavities
}

\author{
Cyril Petitjean, ${ }^{1}$ Daniel Waltner, ${ }^{1}$ Jack Kuipers, ${ }^{1}$ İnanç Adagideli,,${ }^{1,2}$ and Klaus Richter ${ }^{1}$ \\ ${ }^{1}$ Institut für Theoretische Physik, Universität Regensburg, 93040 Regensburg, Germany \\ ${ }^{2}$ Faculty of Engineering and Natural Sciences, Sabanci University, Tuzla, 34956 Istanbul, Turkey \\ (Received 9 June 2009; revised manuscript received 7 August 2009; published 10 September 2009)
}

\begin{abstract}
We address frequency-dependent quantum transport through mesoscopic conductors in the semiclassical limit. By generalizing the trajectory-based semiclassical theory of dc quantum transport to the ac case, we derive the average screened conductance as well as ac weak-localization corrections for chaotic conductors. Thereby we confirm respective random matrix results and generalize them by accounting for Ehrenfest time effects. We consider the case of a cavity connected through many leads to a macroscopic circuit which contains ac sources. In addition to the reservoir the cavity itself is capacitively coupled to a gate. By incorporating tunnel barriers between cavity and leads we obtain results for arbitrary tunnel rates. Finally, based on our findings we investigate the effect of dephasing on the charge relaxation resistance of a mesoscopic capacitor in the linear low-frequency regime.
\end{abstract}

DOI: $10.1103 /$ PhysRevB.80.115310

PACS number(s): 05.45.Mt, 74.40.+k, 73.23.- b, 03.65.Yz

\section{INTRODUCTION}

In contrast to dc-transport experiments, the applied external frequency $\omega$ of an ac-driven mesoscopic structure provides a new energy scale $\hbar \omega$ that permits one to access further properties of these systems, including their intrinsic charge distribution and dynamics. The experimental interest in the ac response of mesoscopic conductors goes back to the work of Pieper and Price ${ }^{1}$ on the dynamic conductance of a mesoscopic Aharonov-Bohm ring. This pioneering work was followed by several experiments ranging from photonassisted transport to quantum shot noise..$^{2-7}$ More recently, the ac regime has been experimentally reinvestigated achieving the measurement of the in- and out-of-phase parts of the ac conductance ${ }^{8}$ and the realization of a high-frequency single-electron source. ${ }^{9}$ Moreover, the recent rise of interest in the full counting statistics of charge transfer has led to a reexamination of the frequency noise spectra. ${ }^{10-12}$ This experimental progress has since triggered renewed theoretical interest in time-dependent mesoscopic transport. ${ }^{13-18}$

One way to tackle the ac-transport problem is to start from linear-response theory for a given potential distribution of the sample. ${ }^{19-21}$ This involves the difficulty that, in principle, the potential distribution and more precisely its link to the screening is unknown. Another approach consists of deriving the ac response to an external perturbation that only enters into quantities describing the reservoirs. Such approaches were initiated by Pastawski ${ }^{22}$ within a nonequilibruium Green's function-based generalized LandauerBüttiker formalism and then the scattering matrix formalism of a time-dependent system was developed by Büttiker et $a l{ }^{23,24}$ Since the energy is in general no longer conserved for an ac bias, the formalism is based on the concept of a scattering matrix that depends on two energy arguments ${ }^{25}$ or equivalently on two times. ${ }^{26}$ Fortunately, when the inverse frequency is small compared to the time to escape the cavity, the ac transport can be expressed in terms of the derivative of the scattering matrix with respect to energy. ${ }^{27}$ In this paper we start from the time-dependent scattering-matrix formalism and limit our investigations to open, classically chaotic ballistic conductors in the low-frequency regime. ${ }^{28}$

For ac transport we calculate the average correlator of scattering matrices $\mathbf{S}(E)$ at different energies $E$. For this we need to know the joint distribution of the matrix elements $S_{\alpha \beta ; i j}$ at different values of the energy or other parameters. (We label the reservoirs connected to the conductor by a Greek index and the mode number by a Latin index.) To our knowledge a general solution to this problem does not yet exist for chaotic systems. However, in the limit of a large number of channels, the first moments of the distribution $S_{\alpha \beta ; j}(E) S_{\alpha \beta ; i j}^{\dagger}\left(E^{\prime}\right)$ were derived using both semiclassical methods ${ }^{29,30}$ and various random-matrix-theory(RMT-)based methods. ${ }^{26,31-33}$ Although the ac-transport properties of ballistic chaotic systems seem to be well described by the RMT of transport ${ }^{33}$ of Brouwer and Büttiker, we develop a semiclassical approach for three reasons: first, this allows us to confirm the random matrix prediction by using a complementary trajectory-based semiclassical method. Second, the energy dependence in the random-matrix formalism was introduced by resorting to artificial models such as the "stub model." ${ }^{26,33}$ While being powerful, this treatment is far from microscopic or natural. The third and strongest reason is to go beyond the RMT treatment and investigate the crossover to the classical limit. Similarly as for the static case RMT is not applicable in this regime. As first noticed by Aleiner and Larkin, ${ }^{34}$ ballistic transport is characterized by a new time scale, known as the Ehrenfest time $\tau_{\mathrm{E}},{ }^{35,36}$ that controls the appearance of interference effects. The Ehrenfest time corresponds to the time during which a localized wavepacket spreads to a classical length scale. Typically, in open chaotic systems two such lengths are relevant, the system size $L$ and the lead width $W$. We can thus define an Ehrenfest time associated with each one, ${ }^{37,38}$ the closed-cavity Ehrenfest time

$$
\tau_{\mathrm{E}}^{\mathrm{cl}}=\lambda^{-1} \ln \left[L / \lambda_{\mathrm{F}}\right]
$$

and the open-cavity Ehrenfest time 


$$
\tau_{\mathrm{E}}^{\mathrm{op}}=\lambda^{-1} \ln \left[W^{2} / \lambda_{\mathrm{F}} L\right],
$$

where $\lambda$ is the classical Lyapunov exponent of the cavity.

Although the success of the semiclassical method (beyond the so-called diagonal approximation, see below) to describe quantitatively universal and nonuniversal dc-transport properties is now clearly established, ${ }^{39-50}$ the corresponding semiclassical understanding of frequency-dependent transport is far less developed. Based on an earlier semiclassical evaluation of matrix element sum rules by Wilkinson ${ }^{51}$ and a semiclassical theory of linear-response functions, ${ }^{52}$ a semiclassical approach to the frequency-dependent conductivity within the Kubo formalism led to an expression of the ac (magneto)conductivity, $\sigma(\omega)$ in terms of a trace formula for classical periodic orbits. ${ }^{53}$ Closely related to this evaluation of $\sigma(\omega)$ is the problem of frequency-dependent (infrared) absorption in ballistic mesoscopic cavities which has been treated semiclassically in Ref. 52. Peaks in the absorption could be assigned to resonance effects when the external frequency $\omega$ corresponds to the inverse periods of fundamental periodic orbits in the cavity. Reference 34 contains a first, $\sigma$-model-based approach to weak-localization effects in the ac-Kubo conductivity, where the findings were interpreted in a quasiclassical trajectory picture (beyond the diagonal approximation). We note also that the semiclassical treatment of the product of scattering matrices $\mathbf{S}(E)$ at different energies has been investigated in different context such as the Ericson fluctuations ${ }^{42}$ and the time delay ${ }^{49}$ however without considering the Ehrenfest time dependence.

The outline of this article is as follows: in Sec. II we introduce our model to treat the system of interest namely a quantum dot under ac bias and recall some basic results about conservation laws in presence of a time-dependent field. In Sec. III we present the method used to treat screening, which is based on a self-consistent approach developed by Büttiker et al. ${ }^{24}$ The admittance, i.e., the ac conductance, is then calculated semiclassically for the particular case of strong coupling to the leads (transparent contact) in Sec. IV, where we illustrate our result by treating the time dependence of a pulsed cavity. We generalize the method to cope with arbitrary tunnel rates in Sec. $\mathrm{V}$ and finally we use our general results to investigate dephasing effects on the chargerelaxation resistance of a mesoscopic capacitor in Sec. VI.

\section{THE MODEL}

We consider a ballistic quantum dot, i.e., a twodimensional chaotic cavity coupled to $M$ electron reservoirs via $M$ leads. Each lead $\alpha$ has a width $W_{\alpha}$ and is coupled to the cavity through a tunnel barrier (see Fig. 1). In addition to the treatment of Ref. 46 we assign a particular tunnel probability to each lead mode. The tunnel barrier is thus characterized by a set of transmission probabilities, $\boldsymbol{\Gamma}_{\alpha}$ $=\left\{\Gamma_{\alpha, 1}, \ldots, \Gamma_{\alpha, N_{\alpha}}\right\}$, with $N_{\alpha}$ the maximum mode number of lead $\alpha$. The chaotic dot is additionally capacitively coupled to a gate connected to a reservoir at voltage $\mathcal{U}_{0}(\omega)$, from which a current $\mathcal{I}_{0}(\omega)$ flows. This capacitive coupling with the gate is taken into account via a geometrical capacitance C. $23,33,54$

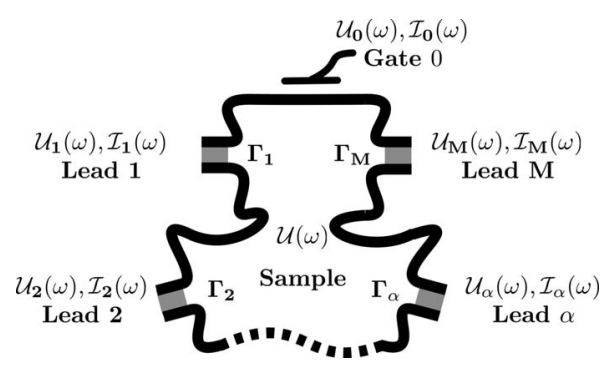

FIG. 1. Two-dimensional chaotic cavity with $M$ leads and one gate 0 . Each lead $\alpha$ has a width $W_{\alpha}$ and is coupled to a reservoir at potential $\mathcal{U}_{\alpha}(\omega)$ and current $\mathcal{I}_{\alpha}(\omega)$. Each tunnel barrier is characterized by the set of transmission probabilities $\boldsymbol{\Gamma}_{\alpha}=\left\{\Gamma_{\alpha, 1}, \ldots, \Gamma_{\alpha, N_{\alpha}}\right\}$. The gate and the sample are capacitively coupled, which leads to a gate current $\mathcal{I}_{0}(\omega)=-i \omega C\left[\mathcal{U}_{0}(\omega)-\mathcal{U}(\omega)\right]$.

We further require that the size of the contact is much smaller than the system size $L$ but still semiclassically large, $1 \ll N_{\alpha} \ll \mathrm{L} / \lambda_{\mathrm{F}}$. This requirement ensures that the particle spend enough time inside the cavity to experience the chaotic dynamics.

As usual for such mesoscopic structures we need to distinguish between quantum and classical time scales. On the quantum side we have already introduced the Ehrenfest times $\left(\tau_{\mathrm{E}}^{\mathrm{op}}, \tau_{\mathrm{E}}^{\mathrm{cl}}\right)$ in Eqs. (1) and (2) while another time scale is the Heisenberg time $\tau_{\mathrm{H}}$, the time to resolve the mean level spacing of the system. On the classical side the time of flight $\tau_{\mathrm{f}}$ between two consecutive bounces at the system cavity wall is relevant. In most ballistic systems or billiards we have $\tau_{\mathrm{f}} \simeq \lambda^{-1}$. Another relevant time scale is the ballistic ergodic time $\tau_{\text {erg }}$ which determines how long it takes for an electron to visit most of the available phase space. However, as we deal with transport properties, a further important time scale is the dwell time $\tau_{\mathrm{D}}$, the average time spent in the cavity before reaching the contact, we have $\tau_{\mathrm{D}} / \tau_{\text {erg }} \gg 1$. The related escape rate therefore satisfies

$$
\tau_{\mathrm{D}}^{-1}=\tau_{\mathrm{H}}^{-1} \sum_{\alpha=1}^{M} \sum_{i=1}^{N_{\alpha}} \Gamma_{\alpha, i} .
$$

For small openings which we consider here, we have $\lambda \tau_{\mathrm{D}}$ $\gg 1$. The ac-transport properties of such a mesoscopic system are characterized by the dimensionless admittance

$$
g_{\alpha \beta}(\omega)=G_{\alpha \beta}(\omega) / G_{0}=G_{0}^{-1} \partial I_{\alpha}(\omega) / \partial U_{\beta}(\omega)
$$

with $G_{0}=d_{s} e^{2} / h$, where $d_{s}=1$ or 2 in the absence or presence of spin degeneracy. In this study we limit ourselves to the coefficients $g_{\alpha \beta}(\omega)$ with $\alpha, \beta=1, \ldots, M$, where the coefficients denoting the gate are determined by current conservation and the freedom to choose the zero point of energy ${ }^{23}$

$$
\sum_{\alpha=0}^{M} g_{\alpha \beta}(\omega)=\sum_{\beta=0}^{M} g_{\alpha \beta}(\omega)=0 .
$$

We note that Eq. (5) is a straightforward consequence of the underlying gauge invariance. Owing to the conservation of charge, the total electric current fulfills the continuity equation 


$$
\boldsymbol{\nabla} \cdot \mathbf{j}_{p}+\frac{\partial \rho}{\partial t}=0
$$

where $\rho$ is the charge density and $\mathbf{j}_{p}$ the particle current density. For dc transport, the charge density is time independent and so we have $\boldsymbol{\nabla} \cdot \mathbf{j}_{p}=0$. Thus the sum of all currents that enter into the dot is always zero. Moreover the current properties must remain unchanged under a simultaneous global shift of the voltages of the reservoirs. These conditions imply the well know unitarity of the scattering matrix ${ }^{55}$

$$
\sum_{\alpha, i} S_{\alpha \beta ; i j}^{\dagger}(E) S_{\alpha \gamma, i k}(E)=\delta_{\beta \gamma ; j k} \text {. }
$$

For ac transport, the product of scattering matrices at different energies no longer obey a similar property, ${ }^{55-58}$ i.e.,

$$
\sum_{\alpha, i} S_{\alpha \beta ; i j}^{\dagger}(E) S_{\alpha \gamma ; i k}\left(E^{\prime}\right) \neq \delta_{\beta \gamma ; j k}
$$

indeed this inequality expresses the fact that due to the possible temporary pile up of charge in the cavity the particle current density no longer satisfies $\boldsymbol{\nabla} \cdot \mathbf{j}_{p}=0$. However one can instead use the Poisson equation

$$
\boldsymbol{\nabla} \cdot \mathbf{D}=\rho,
$$

where $\mathbf{D}=-\boldsymbol{\epsilon} \boldsymbol{\varphi}$ with $\varphi$ the electric potential, to define the total electric-current density which satisfies $\boldsymbol{\nabla} \cdot \mathbf{j}=0$, as a sum of a particle and a displacement current

$$
\mathbf{j}=\mathbf{j}_{p}+\frac{\partial \mathbf{D}}{\partial t} .
$$

In order to find $\mathbf{j}$ one needs to know the electrical field $\mathbf{D}$. In general its calculation is not a trivial task because the intrinsic many-body aspect of the problem makes the treatment of the Poisson Eq. (9) tricky, especially if it is necessary to treat the particle and displacement current on the same footing.

In this work we shall adopt the approach of Ref. 24 to simplify the problem. In this approach the environment is reduced to a single gate, the Coulomb interaction is described by a geometrical capacitance $C$ and the two currents are treated on different footing; the particle current is calculated quantum mechanically via the scattering approach, while the displacement current is treated classically via the electrostatic law [Eqs. (6) and (9)]. This simplification will permit us below to reexpress the Poisson Eq. (9) to obtain the simplest gauge-invariant theory that takes care of the screening. We emphasize that even though our model could be thought of as oversimplified it has the advantage of being able to probe the effects due to the long-range Coulomb interaction. Indeed, for noninteracting particles it is possible to treat the dot and the gate via two sets of uncorrelated continuity equations. The Coulomb interaction removes this possibility and we need to consider the gate and dot as a whole system.

\section{EXPRESSION FOR THE ADMITTANCE}

The method to compute the admittance proceeds in two steps: ${ }^{56}$ first the direct response (particle current) to the change in the external potential is calculated under the assumption that the internal potential $\mathcal{U}(\omega)$ of the sample is fixed. This leads to the definition of the unscreened admittance $g_{\alpha \beta}^{u}(\omega)$. Second, a self-consistent procedure based on the gauge invariance (current conservation and freedom to choose the zero of voltages) is used to obtain the screened admittance $g_{\alpha \beta}(\omega)$.

The unscreened admittance reads ${ }^{23}$

$$
\begin{aligned}
g_{\alpha \beta}^{u}(\omega)= & \int \mathrm{d} E \frac{\left.f\left(E-\frac{\hbar \omega}{2}\right)-f\left(E+\frac{\hbar \omega}{2}\right)\right]}{\hbar \omega} \\
& \times \operatorname{Tr}\left[\delta_{\alpha \beta} \mathbf{1}_{\alpha}-\mathbf{S}_{\alpha \beta}\left(E+\frac{\hbar \omega}{2}\right) \mathbf{S}_{\alpha \beta}^{\dagger}\left(E-\frac{\hbar \omega}{2}\right)\right],
\end{aligned}
$$

where $f(E)$ stands for the Fermi distribution, $\mathbf{S}_{\alpha \beta}$ is the $N_{\alpha}$ $\times N_{\beta}$ scattering matrix from lead $\beta$ to lead $\alpha$, and $\mathbf{1}_{\alpha}$ is an $N_{\alpha} \times N_{\alpha}$ identity matrix. Under the assumption that $\mathcal{U}(\omega)$ is spatially uniform, the screened admittance $g_{\alpha \beta}(\omega)$ is straightforward to obtain. ${ }^{23}$ For sake of completeness we present here only the outline of the method and refer to Ref. 27 for more details.

On the one hand the current response at contact $\alpha$ is

$$
\mathcal{I}_{\alpha}(\omega)=G_{0}\left[\sum_{\beta=1}^{M} g_{\alpha \beta}^{u}(\omega) \mathcal{U}_{\beta}(\omega)+g_{\alpha 0}^{i}(\omega) \mathcal{U}(\omega)\right],
$$

where $g_{\alpha 0}^{i}(\omega)$ is the unknown internal response of the mesoscopic conductor generated by the fluctuating potential $\mathcal{U}(\omega)$. On the other hand the current induced at the gate is

$$
\mathcal{I}_{0}(\omega)=-i \omega C\left[\mathcal{U}_{0}(\omega)-\mathcal{U}(\omega)\right] \text {. }
$$

Gauge invariance permits a shift of $-\mathcal{U}(\omega)$ and provides an expression for the unknown internal response

$$
g_{\alpha 0}^{i}(\omega)=-\sum_{\beta=1}^{M} g_{\alpha \beta}^{u}(\omega) .
$$

Then current conservation, $\sum_{\alpha=1}^{M} \mathcal{I}_{\alpha}(\omega)+\mathcal{I}_{0}(\omega)=0$, yields the result of the screened admittance ${ }^{23}$

$$
g_{\alpha \beta}(\omega)=g_{\alpha \beta}^{u}(\omega)+\frac{\sum_{\delta=1}^{M} g_{\alpha \delta}^{u}(\omega) \sum_{\delta^{\prime}=1}^{M} g_{\delta^{\prime} \beta}^{u}(\omega)}{i \omega C / G_{0}-\sum_{\delta=1}^{M} \sum_{\delta^{\prime}=1}^{M} g_{\delta \delta^{\prime}}^{u}(\omega)} .
$$

In the self-consistent approach used to obtain Eq. (15), the only electron-electron interaction term that has been considered is the capacitive charging energy of the cavity. This implies that we should consider a sufficiently large quantum dot. ${ }^{59}$ We note that, using a $1 / N$ expansion, the selfconsistent approach above was recently formally confirmed in Ref. 60. Moreover, Eq. (15) can be generalized to nonequilibrium problems, using Keldysh nonequilibrium Green's functions. ${ }^{61}$

In the next sections we present the semiclassical evaluation of Eq. (11) in the zero-temperature limit (including finite temperature is straightforward). For reasons of presentation we first give the semiclassical derivation for the transparent case in Sec. IV and then we explore the general case in Sec. 
V. In Sec. VI we present an application of the screened result for tunnel coupling when we compute the relaxation resistance of a mesoscopic chaotic capacitor.

\section{SEMICLASSICAL THEORY FOR THE ADMITTANCE}

\section{A. Semiclassical approximation}

We first consider the multiterminal case assuming transparent barriers, i.e., $\Gamma_{\alpha, i}=1, \forall(\alpha, i)$. In the limit $k_{\mathrm{B}} T \rightarrow 0$ the unscreened admittance, Eq. (11), reduces to

$$
g_{\alpha \beta}^{u}(\omega)=N_{\alpha} \delta_{\alpha \beta}-\operatorname{Tr}\left[\mathbf{S}_{\alpha \beta}\left(E_{\mathrm{F}}+\frac{\hbar \omega}{2}\right) \mathbf{S}_{\alpha \beta}^{\dagger}\left(E_{\mathrm{F}}-\frac{\hbar \omega}{2}\right)\right] .
$$

Semiclassically, the matrix elements for scattering processes from mode $i$ in lead $\beta$ to mode $j$ in lead $\alpha \operatorname{read}^{30,62}$

$$
\begin{aligned}
S_{\alpha \beta ; j i}\left(E_{\mathrm{F}} \pm \frac{\hbar \omega}{2}\right)= & -\int_{\beta} \mathrm{d} x_{0} \int_{\alpha} \mathrm{d} x \frac{\langle j \mid x\rangle\left\langle x_{0} \mid i\right\rangle}{(2 \pi i \hbar)^{1 / 2}} \\
& \times \sum_{\gamma} A_{\gamma} e^{i / \hbar S_{\gamma}\left(x, x_{0} ; E_{\mathrm{F}} \pm \hbar \omega / 2\right)}
\end{aligned}
$$

where $|i\rangle$ is the transverse wave function of the $i$ th mode. Here the $x_{0}$ (or $x$ ) integral is over the cross section of the $\beta$ th (or $\alpha$ th) lead. At this point $\mathbf{S}_{\alpha \beta}$ is given by a sum over classical trajectories, labeled by $\gamma$. The classical paths $\gamma$ connect $\mathbf{X}_{0}=\left(x_{0}, p_{x_{0}}\right)$ (on a cross section of lead $\beta$ ) to $\mathbf{X}=\left(x, p_{x}\right)$ (on a cross section of lead $\alpha$ ). Each path gives a contribution oscillating with action $S_{\gamma}$ (including Maslov indices) evaluated at the energy $E_{\mathrm{F}} \pm \hbar \omega / 2$ and weighted by the complex amplitude $A_{\gamma}$. This reduces to the square root of an inverse element of the stability matrix, ${ }^{63}$ i.e., $A_{\gamma}=\left|\left(\mathrm{d} p_{x_{0}} / \mathrm{d} x\right)_{\gamma}\right|^{1 / 2}$.

We insert Eq. (17) into Eq. (16) and obtain double sums over paths $\gamma$ and $\gamma^{\prime}$, and lead modes $|i\rangle$ and $|j\rangle$. The sum over the channel indices is then performed with the semiclassical approximation, ${ }^{46} \sum_{i=1}^{N_{\beta}}\left\langle x_{0} \mid i\right\rangle\left\langle i \mid x_{0}^{\prime}\right\rangle \approx \delta\left(x_{0}^{\prime}-x_{0}\right)$ and yields

$$
g_{\alpha \beta}^{u}(\omega)-N_{\alpha} \delta_{\alpha \beta}=-\int_{\beta} \mathrm{d} x_{0} \int_{\alpha} \mathrm{d} x \sum_{\gamma, \gamma^{\prime}} \frac{A_{\gamma} A_{\gamma^{\prime}}^{*}}{2 \pi \hbar} e^{i / \hbar \delta S\left(E_{\mathrm{F}}, \omega\right)} .
$$

Here,

$$
\delta S\left(E_{\mathrm{F}}, \omega\right)=S_{\gamma}\left(x_{0}, x ; E_{\mathrm{F}}+\frac{\hbar \omega}{2}\right)-S_{\gamma^{\prime}}\left(x_{0}, x ; E_{\mathrm{F}}-\frac{\hbar \omega}{2}\right) .
$$

As we are interested in the limit $\hbar \omega \ll E_{\mathrm{F}}$, we can expand $\delta S\left(E_{\mathrm{F}}, \omega\right)$ around $E_{\mathrm{F}}$. The dimensionless ac conductance is then given by

$$
\begin{aligned}
g_{\alpha \beta}^{u}(\omega)-N_{\alpha} \delta_{\alpha \beta}= & -\int_{\beta} \mathrm{d} x_{0} \int_{\alpha} \mathrm{d} x \sum \frac{A \gamma_{\gamma, \gamma^{\prime}}^{*}}{2 \pi \hbar} \\
& \times \exp \left[\frac{i}{\hbar} \delta S\left(E_{\mathrm{F}}\right)+\frac{i \omega}{2}\left(t_{\gamma}+t_{\gamma^{\prime}}\right)\right]
\end{aligned}
$$

where $\delta S\left(E_{\mathrm{F}}\right)=S_{\gamma}\left(x_{0}, x ; E_{\mathrm{F}}\right)-S_{\gamma^{\prime}}\left(x_{0}, x ; E_{\mathrm{F}}\right)$ and $t_{\gamma}\left(t_{\gamma^{\prime}}\right)$ is the total duration of the path $\gamma\left(\gamma^{\prime}\right)$. Equation (20) is the starting point of our further investigations.

\section{B. Drude Admittance}

We are interested in quantities arising from averaging over variations in the energy or cavity shapes. For most sets of paths, the phase given by the linearized action difference $\delta S\left(E_{\mathrm{F}}\right)$ will oscillate widely with these variations, so their contributions will average out. In the semiclassical limit, the dominant contribution to Eq. (20) is the diagonal one, $\gamma$ $=\gamma^{\prime}$, which leads to $t_{\gamma}=t_{\gamma^{\prime}}, \delta S\left(E_{\mathrm{F}}\right)=0$ and gives

$$
g_{\alpha \beta}^{u, \mathrm{D}}(\omega)=N_{\alpha} \delta_{\alpha \beta}-\int_{\beta} \mathrm{d} x_{0} \int_{\alpha} \mathrm{d} x \sum_{\gamma} \frac{\left|A_{\gamma}\right|^{2}}{2 \pi \hbar} e^{i \omega t_{\gamma}} .
$$

In the following we proceed along the lines of Ref. 43. The key point is the replacement of the semiclassical amplitudes by their corresponding classical probabilities. To this end we use a classical sum rule valid under ergodic assumptions ${ }^{64}$

$$
\begin{aligned}
\sum_{\gamma}\left|A_{\gamma}\right|^{2} e^{i \omega t} \gamma[\cdots]_{\gamma}= & \int_{0}^{\infty} \mathrm{d} t \int_{-\pi / 2}^{\pi / 2} \mathrm{~d} \theta_{0} \mathrm{~d} \theta e^{i \omega t} p_{\mathrm{F}} \cos \left(\theta_{0}\right) \\
& \times P\left(\mathbf{X}, \mathbf{X}_{0} ; t\right)[\cdots]_{\mathbf{X}_{0}}
\end{aligned}
$$

In Eq. (22), $p_{\mathrm{F}} \cos \left(\theta_{0}\right)$ is the initial momentum along the injection lead and $P\left(\mathbf{X}, \mathbf{X}_{0} ; t\right)$ the classical probability density to go from an initial phase-space point $\mathbf{X}_{0}=\left(x_{0}, \theta_{0}\right)$ at the boundary between the system and the lead to the corresponding point $\mathbf{X}=(x, \theta)$. The average of $P$ over an ensemble or over energy gives a smooth function that reads

$$
\left\langle P\left(\mathbf{X}, \mathbf{X}_{0} ; t\right)\right\rangle=\frac{\cos (\theta)}{2 \tau_{\mathrm{D}} \Sigma_{\alpha=1}^{M} W_{\alpha}} e^{-t / \tau_{\mathrm{D}}}
$$

with the escape rate $\tau_{\mathrm{D}}^{-1}$ given in Eq. (3).

Using Eqs. (21)-(23), we recover the Drude admittance ${ }^{33}$

$$
g_{\alpha \beta}^{u, \mathrm{D}}(\omega)=N_{\alpha} \delta_{\alpha \beta}-\frac{N_{\alpha} N_{\beta}}{N}\left(\frac{1}{1-i \omega \tau_{\mathrm{D}}}\right),
$$

where $N=\sum_{\alpha=1}^{M} N_{\alpha}$.

\section{Weak localization for transmission, reflection, and coherent backscattering}

\section{Weak localization}

The leading-order weak-localization correction to the conductance was identified in Refs. 34 and 40 as those arising from trajectories that are exponentially close almost everywhere except in the vicinity of an encounter. An example of 


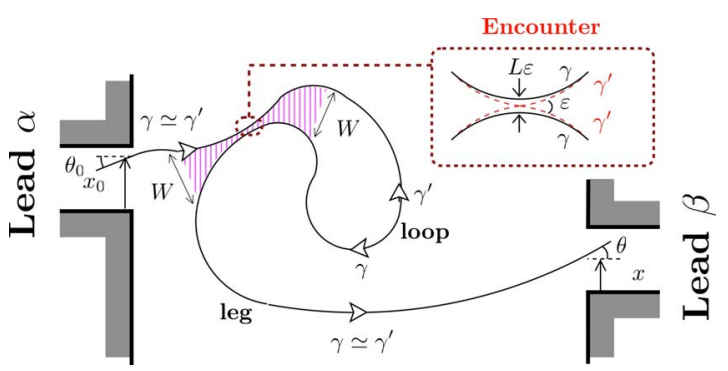

FIG. 2. (Color online) A semiclassical contribution to weak localization for a system with strong (transparent) coupling to the leads. The two paths follow each other closely everywhere except at the encounter, where one path (dashed line) crosses itself at an angle $\epsilon$, while the other one (full line) does not (going the opposite way around the loop). The cross-hatched area denotes the region where two segments of the solid paths are paired (within $W_{\alpha}$ $\simeq W_{\beta} \simeq W$ of each other)

such a trajectory pair for chaotic ballistic systems is shown in Fig. 2. At the encounter, separating the "loop" from the "legs," one of the trajectories $\left(\gamma^{\prime}\right)$ intersects itself, while the other one $(\gamma)$ avoids the crossing. Thus, they travel along the loop they form in opposite directions. In the semiclassical limit, only pairs of trajectories with a small crossing angle $\epsilon$ contribute significantly to weak localization. In this case, each trajectory remains correlated for some time on both sides of the encounter. In other words, the smallness of $\epsilon$ requires two minimal times: $T_{\mathrm{L}}(\epsilon)$ to form a loop and $T_{\mathrm{W}}(\epsilon)$ in order for the legs to separate before escaping into different leads. The encounter introduces a typical length scale $\delta r_{\perp}$ that corresponds to the perpendicular distance between the two paths in the vicinity of the encounter. In the case of hyperbolic dynamics, we get $\delta r_{\perp}=v_{\mathrm{F}} \epsilon /(2 \lambda) \sim L \epsilon$. Hence, the typical minimal time is given by $T_{\ell}(\epsilon)=\lambda^{-1} \ln \left[\left(\ell / \delta r_{\perp}\right)^{2}\right]$ with $\ell=\{\mathrm{L}, \mathrm{W}\}$ that we can approximate as

$$
\begin{gathered}
T_{\mathrm{L}}(\epsilon) \simeq \lambda^{-1} \ln \left[\epsilon^{-2}\right], \\
T_{\mathrm{W}}(\epsilon) \simeq \lambda^{-1} \ln \left[\epsilon^{-2}(W / L)^{2}\right] .
\end{gathered}
$$

The presence of the external driving does not change this picture. Each weak-localization contribution accumulates a phase difference given by the linearized action $\delta S\left(E_{\mathrm{F}}\right)$ $\simeq \delta S_{\mathrm{RS}}=E_{\mathrm{F}} \epsilon^{2} / \lambda .{ }^{40}$ Following the same lines as for the derivation of the Drude contribution, though the sum over paths is now restricted to paths with an encounter, the sum rule (22) still applies, provided the probability $P\left(\mathbf{X}, \mathbf{X}_{0} ; t\right)$ is restricted to paths which cross themselves. To ensure this we write

$$
\begin{aligned}
P\left(\mathbf{X}, \mathbf{X}_{0} ; t\right)= & \int_{\mathcal{C}} \mathrm{d} \mathbf{R}_{2} \mathrm{~d} \mathbf{R}_{1} P\left(\mathbf{X}, \mathbf{R}_{2} ; t-t_{2}\right) \\
& \times P\left(\mathbf{R}_{2}, \mathbf{R}_{1} ; t_{2}-t_{1}\right) P\left(\mathbf{R}_{1}, \mathbf{X}_{0} ; t_{1}\right),
\end{aligned}
$$

where the integration is performed over the energy surface $\mathcal{C}$. Here, we use $\mathbf{R}_{i}=\left(\mathbf{r}_{i}, \phi_{i}\right), \phi_{i} \in[-\pi, \pi]$ for phase-space points inside the cavity, while $\mathbf{X}$ lies on the lead surface as before.
We then restrict the probabilities inside the integral to trajectories which cross themselves at phase-space positions $\mathbf{R}_{1,2}$ with the first (or second) visit of the crossing occurring at time $t_{1}$ (or $t_{2}$ ). We can write $\mathrm{d} \mathbf{R}_{2}=v_{\mathrm{F}}^{2} \sin \epsilon \mathrm{d} t_{1} \mathrm{~d} t_{2} \mathrm{~d} \epsilon$ and set $\mathbf{R}_{2}=\left(\mathbf{r}_{1}, \phi_{1} \pm \epsilon\right)$. Then the weak-localization correction is given by

$$
g_{\alpha \beta}^{u, \mathrm{wl}}(\omega)=\frac{1}{\pi \hbar} \int_{\beta} \mathrm{d} \mathbf{X}_{0} \int \mathrm{d} \epsilon \mathfrak{R e}\left[e^{i \delta S_{\mathrm{RS}} / \hbar}\right]\left\langle F\left(\mathbf{X}_{0}, \epsilon, \omega\right)\right\rangle,
$$

with

$$
\begin{aligned}
F\left(\mathbf{X}_{0}, \epsilon, \omega\right)= & 2 v_{\mathrm{F}}^{2} \sin \epsilon \int_{T_{\mathrm{L}}+T_{\mathrm{W}}}^{\infty} \mathrm{d} t \int_{T_{\mathrm{L}}+T_{\mathrm{W}} / 2}^{t-T_{\mathrm{W}} / 2} \mathrm{~d} t_{2} \int_{T_{\mathrm{W}} / 2}^{t_{2}-T_{\mathrm{L}}} \mathrm{d} t_{1} \\
& \times p_{\mathrm{F}} \cos \theta_{0} \int_{\mathrm{R}} \mathrm{d} \mathbf{Y} \int_{\mathcal{C}} \mathrm{d} \mathbf{R}_{1} P\left(\mathbf{X}, \mathbf{R}_{2} ; t-t_{2}\right) \\
& \times P\left(\mathbf{R}_{2}, \mathbf{R}_{1} ; t_{2}-t_{1}\right) P\left(\mathbf{R}_{1}, \mathbf{X}_{0} ; t_{1}\right) e^{i \omega t}
\end{aligned}
$$

Under our approximation $t_{\gamma^{\prime}} \simeq t_{\gamma}=t$, the introduction of the driving frequency leads to performing a Fourier transform of the survival probability and we obtain

$$
\begin{aligned}
\left\langle F\left(\mathbf{X}_{0}, \epsilon, \omega\right)\right\rangle= & \frac{\left(v_{\mathrm{F}} \tau_{\mathrm{D}}\right)^{2} p_{\mathrm{F}} \sin \epsilon \cos \theta_{0}}{\pi \Omega} \frac{N_{\alpha}}{N} \\
& \times \frac{\exp \left[-T_{\mathrm{L}} / \tau_{\mathrm{D}}\right] \exp \left[i \omega\left(T_{\mathrm{L}}+T_{\mathrm{W}}\right)\right]}{\left(1-i \omega \tau_{\mathrm{D}}\right)^{3}}
\end{aligned}
$$

with $\Omega$ the cavity area. Inserting Eq. (29) into Eq. (27), the $\epsilon$ integral is dominated by small-angle $(\epsilon \ll 1)$ contributions, allowing for the approximation $\sin \epsilon \simeq \epsilon$ and pushing the upper limit to infinity. This yields an Euler Gamma function times an exponential term $e^{-\tau_{\mathrm{E}}^{\mathrm{cl}} / \tau_{\mathrm{D}}} e^{i \omega\left(\tau_{\mathrm{E}}^{\mathrm{cl}}+\tau_{\mathrm{E}}^{\mathrm{op}}\right)}$ (with $\tau_{\mathrm{E}}^{\mathrm{op}}$ and $\tau_{\mathrm{E}}^{\mathrm{cl}}$ given by Eqs. (1) and (2) that reads, to leading order in $\left(\lambda \tau_{\mathrm{D}}\right)^{-1}$

$$
\begin{aligned}
& \int_{0}^{\infty} \mathrm{d} \epsilon 2 \Re \mathrm{Re}\left[\exp \left(\frac{i E_{\mathrm{F}} \epsilon^{2}}{\lambda \hbar}\right)\right] \epsilon^{1+2 / \lambda \tau_{\mathrm{D}}\left(1-2 i \omega \tau_{\mathrm{D}}\right)}\left(\frac{\mathrm{W}}{\mathrm{L}}\right)^{2 i \omega / \lambda} \\
& \simeq-\frac{\pi \hbar}{m v_{\mathrm{F}}^{2} \tau_{\mathrm{D}}} e^{-\tau_{\mathrm{E}}^{\mathrm{cl}} / \tau_{\mathrm{D}}+i \omega\left(\tau_{\mathrm{E}}^{\mathrm{cl}}+\tau_{\mathrm{E}}^{\mathrm{op}}\right)}\left(1-2 i \omega \tau_{\mathrm{D}}\right) \\
& +\mathcal{O}\left[\frac{1}{\lambda \tau_{\mathrm{D}}}\right] \text {. }
\end{aligned}
$$

Performing the $\mathbf{X}_{0}$ integral and using $N_{\beta}=(\pi \hbar)^{-1} p_{\mathrm{F}} W_{\beta}$ and $N=\left(\hbar \tau_{\mathrm{D}}\right)^{-1} m \Omega$, the weak-localization correction to the unscreened admittance is

$$
g_{\alpha \beta}^{u, \mathrm{wl}}(\omega)=\frac{N_{\alpha} N_{\beta}}{N^{2}} e^{-\tau_{\mathrm{E}}^{\mathrm{cl}} / \tau_{\mathrm{D}}} \frac{\left(1-2 i \omega \tau_{\mathrm{D}}\right) e^{i \omega\left(\tau_{\mathrm{E}}^{\mathrm{cl}}+\tau_{\mathrm{E}}^{\mathrm{op}}\right)}}{\left(1-i \omega \tau_{\mathrm{D}}\right)^{3}} .
$$

We note that due to the absence of unitarity of the unscreened admittance we need to explicitly evaluate all the elements of $g_{\alpha \beta}^{u}(\omega)$. The weak-localization contribution to reflection $r_{\alpha \alpha}^{u, \mathrm{wl}}(\omega)$ is derived in the same manner as $g_{\alpha \beta}^{u, \mathrm{wl}}(\omega)$, replacing however the factor $N_{\beta} / N$ by $N_{\alpha} / N$. We then obtain 


$$
r_{\alpha \alpha}^{u, \mathrm{wl}}(\omega)=\left(\frac{N_{\alpha}}{N}\right)^{2} e^{-\tau_{\mathrm{E}}^{\mathrm{cl}} / \tau_{\mathrm{D}}} \frac{\left(1-2 i \omega \tau_{\mathrm{D}}\right) e^{i \omega\left(\tau_{\mathrm{E}}^{\mathrm{cl}}+\tau_{\mathrm{E}}^{\mathrm{op}}\right)}}{\left(1-i \omega \tau_{\mathrm{D}}\right)^{3}}
$$

However as in the dc case there is another leading-order contribution to the reflection, the so-called coherent backscattering. This differs from weak localization as the path segments that hit the lead are correlated. This mechanism should be treated separately when computing the Ehrenfest time dependence, which is the object of the next paragraph.

\section{Coherent backscattering}

Though the correlation between two paths does not influence the treatment of the external frequency, it induces an action difference $\delta S\left(E_{\mathrm{F}}\right)=\delta S_{\mathrm{cbs}}=-\left(p_{0 \perp}+m \lambda r_{0 \perp}\right) r_{0 \perp}$ where the perpendicular difference in position and momentum are $r_{0 \perp}=\left(x_{0}-x\right) \cos \theta_{0}$ and $p_{0 \perp}=-p_{\mathrm{F}}\left(\theta-\theta_{0}\right)$. As for weak localization, we can identify two time scales, $\frac{1}{2} T_{\mathrm{L}}^{\prime}$ and $\frac{1}{2} T_{\mathrm{W}}^{\prime}$, associated with the time for paths to spread to $L$ and $W$, respectively. However unlike for weak localization we define these time scales as times measured from the lead rather than from the encounter. Thus we have

$$
T_{\ell}^{\prime}\left(r_{0 \perp}, p_{0 \perp}\right) \simeq \frac{2}{\lambda} \ln \left[(m \lambda \ell) /\left|p_{0 \perp}+m \lambda r_{0 \perp}\right|\right],
$$

with $\ell=\{L, W\}{ }^{48}$ Replacing the integral over $\mathbf{X}_{0}$ by an integral over $\left(r_{0 \perp}, p_{0 \perp}\right)$ and using $p_{\mathrm{F}} \cos \theta_{0} \mathrm{~d} \mathbf{X}_{0}=\mathrm{d} p_{0 \perp} \mathrm{d} r_{0 \perp}$, the coherent-backscattering contribution reads

$$
r_{\alpha \alpha}^{u, \mathrm{cbs}}(\omega)=(\pi \hbar)^{-1} \int_{\alpha} \mathrm{d} p_{0 \perp} \mathrm{d} r_{0 \perp} \mathfrak{R e}\left[e^{i / \hbar \delta S_{\mathrm{cbs}}}\right]\left\langle F^{\mathrm{cbs}}\left(\mathbf{X}_{0}, \omega\right)\right\rangle
$$

with

$$
\begin{aligned}
\left\langle F^{\mathrm{cbs}}\left(\mathbf{X}_{0}, \omega\right)\right\rangle & =\int_{T_{\mathrm{L}}^{\prime}}^{\infty} \mathrm{d} t \int_{\alpha} \mathrm{d} \mathbf{X} P\left(\mathbf{X}, \mathbf{X}_{0} ; t\right) e^{i \omega t} \\
& =\frac{N_{\alpha}}{N} \frac{e^{-\left(T_{\mathrm{L}}^{\prime}-1 / 2 T_{\mathrm{W}}^{\prime}\right) / \tau_{\mathrm{D}} e^{i \omega T_{\mathrm{L}}^{\prime}}}}{1-i \omega \tau_{\mathrm{D}}}
\end{aligned}
$$

As in the dc case we perform a change in variables $\tilde{p}_{0 \perp}$ $=p_{0 \perp}+m \lambda r_{0 \perp}$. Then we push the $\tilde{p}_{0 \perp}$ integral limit to infinity and evaluate the $r_{0 \perp}$ integral over $W_{\alpha}$. This result

$$
\begin{gathered}
\int_{-\infty}^{\infty} \mathrm{d} \tilde{p}_{0 \perp} \frac{\hbar \sin \left(\widetilde{p}_{0 \perp} W_{\alpha} / \hbar\right)}{\widetilde{p}_{0 \perp}}\left|\frac{\tilde{p}_{0 \perp}}{m \lambda L}\right|^{\left(1-2 i \omega \tau_{\mathrm{D}}\right) / \lambda \tau_{\mathrm{D}}}\left(\frac{W}{L}\right)^{1 / \lambda \tau_{\mathrm{D}}} \\
=\pi \hbar e^{-\tau_{\mathrm{E}}^{\mathrm{cl}} / \tau_{\mathrm{D}}} e^{i \omega\left(\tau_{\mathrm{E}}^{\mathrm{cl}}+\tau_{\mathrm{E}}^{\mathrm{op}}\right)}+\mathcal{O}\left[\left(\lambda \tau_{\mathrm{D}}\right)^{-1}\right]
\end{gathered}
$$

together with Eqs. (34) and (35) yields

$$
r_{\alpha \alpha}^{u, \mathrm{cbs}}(\omega)=-\frac{N_{\alpha}}{N} e^{-\tau_{\mathrm{E}}^{\mathrm{cl}} / \tau_{\mathrm{D}}} \frac{e^{i \omega\left(\tau_{\mathrm{E}}^{\mathrm{cl}}+\tau_{\mathrm{E}}^{\mathrm{op}}\right)}}{\left(1-i \omega \tau_{\mathrm{D}}\right)} .
$$

Surprisingly the coherent-backscattering contribution thus has exactly the same exponential dependence on $\tau_{\mathrm{E}}^{\mathrm{op}}$ and $\tau_{\mathrm{E}}^{\mathrm{cl}}$ as the other weak-localization contributions. While in the dc case this property is a consequence of current conservation, this fact is not obvious in the ac case.
At this point we can summarize our results for the unscreened admittance. From Eqs. (24), (31), (32), and (37), $\left\langle g_{\alpha \beta}^{u}(\omega)\right\rangle$ can be written as

$$
\begin{aligned}
\left\langle g_{\alpha \beta}^{u}(\omega)\right\rangle= & \delta_{\alpha \beta} N_{\alpha}-\frac{N_{\alpha} N_{\beta}}{N\left(1-i \omega \tau_{\mathrm{D}}\right)} \\
& +\frac{N_{\alpha} \exp \left[-\frac{\tau_{\mathrm{E}}^{\mathrm{cl}}}{\tau_{\mathrm{D}}}\right] \exp \left[i \omega\left(\tau_{\mathrm{E}}^{\mathrm{cl}}+\tau_{\mathrm{E}}^{\mathrm{op}}\right)\right]}{N\left(1-i \omega \tau_{\mathrm{D}}\right)} \\
& \times\left(\frac{N_{\beta}\left(1-2 i \omega \tau_{\mathrm{D}}\right)}{N\left(1-i \omega \tau_{\mathrm{D}}\right)^{2}}-\delta_{\alpha \beta}\right)+\mathcal{O}\left(N^{-1}\right) .
\end{aligned}
$$

First we note that in the limit of zero Ehrenfest time we recover the RMT result for the unscreened admittance of Brouwer and Büttiker. ${ }^{33}$ Concerning the Ehrenfest time dependence of the admittance, we note that the result is consistent with the absorption study performed in Ref. 65. As for the dc case we find the absence of the Ehrenfest time $\tau_{\mathrm{E}}^{\mathrm{op}}$ in the term $\exp \left[-\tau_{\mathrm{E}}^{\mathrm{cl}} / \tau_{\mathrm{D}}\right]$ which derives from the classical correlation between the paths that constitute the encounter. The physical origin of the term $\exp \left[i \omega\left(\tau_{\mathrm{E}}^{\mathrm{cl}}+\tau_{\mathrm{E}}^{\mathrm{op}}\right)\right]$ comes from the fact that both trajectories that contribute to weak localization and coherent backscattering involve an encounter that has a minimal duration of $\left(\tau_{\mathrm{E}}^{\mathrm{cl}}+\tau_{\mathrm{E}}^{\mathrm{op}}\right)$ (leg part and loop part of the encounter, see Fig. 2). The presence of this minimal duration, $2 \tau_{\mathrm{E}}^{\mathrm{e}}=\tau_{\mathrm{E}}^{\mathrm{cl}}+\tau_{\mathrm{E}}^{\mathrm{op}}$, is in accordance with the Ehrenfest time-shift prediction of the quantum correction to the survival probability ${ }^{66}$ and the photofragmentation statistics. ${ }^{67}$ We return to the Ehrenfest time dependence in Sec. IV E.

We can also consider the effect of a magnetic flux on the mesoscopic admittance. A weak magnetic field has little effect on the classical dynamics but generates a phase difference between two trajectories that travel in opposite directions around a weak-localization generating closed loop. This phase difference is $\Phi / \Phi_{0}$, where $\Phi_{0}$ is the flux quantum, and $\Phi$ is proportional to the flux through the directed area enclosed by the loop. To incorporate this in the previous semiclassical treatment we must introduce a factor $\exp \left[i \Phi / \Phi_{0}\right]$ into $F$ in Eq. (29) and $F^{\mathrm{cbs}}$ in Eq. (35). The calculation gives a Lorentzian shape $\mathrm{e}^{30,40,43}$ for the $\Phi$ dependence of the quantum correction to the average admittance

$$
g_{\alpha \beta}^{u, \mathrm{wl} / \mathrm{cbs}}(\omega, \Phi)=\frac{g_{\alpha \beta}^{u, \mathrm{wl} / \mathrm{cbs}}(\omega, 0)}{1+\mathcal{A}^{2} \Phi^{2}\left(\tau_{\mathrm{f}} / \tau_{\mathrm{D}}-i \omega \tau_{\mathrm{f}}\right)^{-1}} .
$$

Here $\mathcal{A}^{2}=\alpha \Omega^{2}$, with $\alpha$ a system-dependent parameter of order unity, $\Omega$ is the cavity area and $\tau_{\mathrm{f}}$ is the time of flight between two consecutive bounces at the cavity wall.

\section{The screened admittance}

Following the self-consistent approach, the screened admittance is straightforwardly obtained when we substitute Eq. (38) into Eq. (15) and expand the result to leading order in $N^{-1}$. This simple substitution is justified because the typical fluctuations of the unscreened admittance are of order $N^{-2}$. The screened admittance then reads 


$$
\begin{aligned}
\left\langle g_{\alpha \beta}(\omega)\right\rangle= & \delta_{\alpha \beta} N_{\alpha}-\frac{N_{\alpha} N_{\beta}}{N(1-i \omega \tau)} \\
& +\frac{N_{\alpha} \exp \left[-\frac{\tau_{\mathrm{E}}^{\mathrm{cl}}}{\tau_{\mathrm{D}}}\right] \exp \left[i \omega\left(\tau_{\mathrm{E}}^{\mathrm{cl}}+\tau_{\mathrm{E}}^{\mathrm{op}}\right)\right]}{N\left(1-i \omega \tau_{\mathrm{D}}\right)} \\
& \times\left(\frac{N_{\beta}(1-2 i \omega \tau)}{N(1-i \omega \tau)^{2}}-\delta_{\alpha \beta}\right)+\mathcal{O}\left(N^{-1}\right),
\end{aligned}
$$

where $\tau^{-1}=\tau_{\mathrm{D}}^{-1}+N G_{0} / C$ is the charge-relaxation time or quantum RC time. Equation (40) is the first intermediate result from which we can draw some general conclusions. At zero Ehrenfest time we recover the two-terminal result of Brouwer and Büttiker in Ref. 33. The comparison between the screened [Eq. (40)] and unscreened [Eq. (38)] admittance shows that the screening amounts to the replacement of the dwell time $\tau_{\mathrm{D}}$ by the RC time $\tau$ everywhere up to the prefactor of the third term. Only for the weak localization and the coherent-backscattering contributions does the dwell time-dependence survive. Though the relevant time scale for the classical admittance is the charge-relaxation time $\tau$, the quantum corrections are characterized by the dwell time $\tau_{\mathrm{D}}$. It is important to remember that $\tau_{\mathrm{D}}$ is a characteristic time scale of the noninteracting system. Its relevance here has its origin in the fact that weak localization is due to the interference of electronic waves, which is unimportant for charge accumulation in the system. We recall that, as constructed in the framework of the model, the admittance matrix Eq. (40) is current conserving if the gate is included. The elements of the admittance related to the gate are obtained via the sum rule (5). Nevertheless, if we impose this above sum rule to the unscreened result we also obtain a conserved current and this situation corresponds to a cavity which has infinite capacitance to the gate. In the reverse limit of zero capacitance we reach the charge-neutral regime that corresponds to putting $\tau=0$ in Eq. (40). Upon performing that, we recover the charge-neutral limit obtained by Aleiner and Larkin in Refs. 34 and 68 which for the conventional weak-localization contribution reads

$$
g_{\alpha \beta}^{\mathrm{wl}, \tau=0}(\omega)=\frac{N_{\alpha} N_{\beta}}{N^{2}} \frac{\exp \left[-\frac{\tau_{\mathrm{E}}^{\mathrm{cl}}}{\tau_{\mathrm{D}}}+i \omega\left(\tau_{\mathrm{E}}^{\mathrm{cl}}+\tau_{\mathrm{E}}^{\mathrm{op}}\right)\right]}{\left(1-i \omega \tau_{\mathrm{D}}\right)} .
$$

We note that for the particular geometry of a capacitor (only one lead and one gate) since Eq. (40) is valid for any capacitive coupling, we can obtain the effect of the Ehrenfest time scale on the interference correction to the admittance of a mesoscopic capacitor. This was not possible within the charge-neutral limit approach of Aleiner and Larkin since the interference corrections considered here are absent in that case.

Here one important remark is due. In both, Eqs. (40) and (41) the admittance involves an oscillatory behavior as a function of the Ehrenfest time, which should in principle be more easily accessible experimentally. Indeed, we see here in our quest for the Ehrenfest time physics a clear advantage in investigating weak localization in the ac regime. In the static case, the ratio $\tau_{\mathrm{E}} / \tau_{\mathrm{D}}$ is the only relevant and tunable parameter for the dc weak-localization correction. Consequently, the range of experimental investigation is considerably reduced by the logarithmic dependence of $\tau_{\mathrm{E}}$ on the system size. For the dynamical weak localization the frequency dependence $\omega$ combined with the capacitive coupling $C$ provides more freedom in probing $\tau_{\mathrm{E}}$ behavior. However, although the $\omega \tau_{\mathrm{E}}$ Ehrenfest time dependence was predicted in Ref. 34 (in which some possible experimental verification was forecasted in a magnetoconductance experiment or in an optical backscattering experiment), we are not aware of any experimental verification of the existence of such an oscillation. To the date there exist only two experiments devoted to exploring the $\tau_{\mathrm{E}}$ signature: The shot-noise experiment by Oberholzer et al. ${ }^{69}$ and the weak-localization experiment in an antidot lattice by Yevtushenko et al. ${ }^{70}$ Both experiments were performed in the static case.

To estimate the typical time, respectively, frequency scales for observing the oscillatory Ehrenfest time effects in the ac regime, consider a ballistic cavity based on a highmobility two-dimensional electron gas (2DEG) built from GaAs. For a cavity size of about $\mathrm{L}=5 \mu \mathrm{m}$, a Fermi wavelength $\lambda_{\mathrm{F}}=30 \mathrm{~nm}$, and assuming a Lyapunov exponent on the order of the inverse time of flight through the cavity, that is, $v_{\mathrm{F}} / L \approx 5 \times 10^{9} \mathrm{sec}^{-1}$, we can estimate the Ehrenfest time scale to be on the order of nanoseconds. This corresponds to Gigahertz frequencies that require techniques already established in quantum-dot experiments.

\section{E. Pulsed cavities}

In this section we comment on the Ehrenfest time dependence of the admittance and its link to that of the survival probability. ${ }^{66,67}$ To this end we consider the particular case of a pulsed cavity, ${ }^{58}$ i.e., the application of a pulse $\mathcal{U}_{\alpha}(t)$ $=a_{\alpha} \delta(t)$ to one of the contacts $\alpha$. The response current at contact $\beta$ to such a pulse will be proportional to the frequency integral over the ac conductance

$$
g_{\alpha \beta}^{u}(t)=\frac{1}{2 \pi} \int \mathrm{d} \omega g_{\alpha \beta}^{u}(\omega) \exp (-i \omega t) .
$$

This problem was previously addressed in Ref. 58 where the connection between the RMT calculation of the admittance and RMT results for the quantum and the classical survival probability ${ }^{71,72}$ were discussed. More precisely, in Refs. 71 and 72 a difference between the quantum and the classical survival probability was predicted for times of order $t^{*}$ $=\sqrt{\tau_{\mathrm{D}} \tau_{\mathrm{H}}}$. The conclusion of Ref. 58 was twofold: first, based on the weak-localization correction, a deviation in the unscreened admittance at $t^{*}$ was confirmed while second the screened system was shown not to exhibit such a $t^{*}$ dependence.

Based on our semiclassical results [Eqs. (38) and (40)] we are able to confirm this dependence. For the unscreened admittance, the weak-localization and coherent-backscattering contribution, $\delta g_{\alpha \beta}^{u}(t)=g_{\alpha \beta}^{u, \mathrm{wl}}(t)+g_{\alpha \beta}^{u, \mathrm{cbs}}(t)$, yield a complicated time dependence and read on a log scale 


$$
\begin{aligned}
\ln \left[\frac{N \tau_{\mathrm{D}}}{N_{\alpha} N_{\beta}} \delta g_{\alpha \beta}^{u}(t)\right]= & -\frac{t-\tau_{\mathrm{E}}^{\mathrm{op}}}{\tau_{\mathrm{D}}}+\ln \left[-\frac{\delta_{\alpha \beta}}{N_{\alpha}}+\frac{1}{N}\left(\frac{t-2 \tau_{\mathrm{E}}^{\mathrm{e}}}{\tau_{\mathrm{D}}}\right)\right. \\
& \left.\times\left(2-\frac{t-2 \tau_{\mathrm{E}}^{\mathrm{e}}}{2 \tau_{\mathrm{D}}}\right)\right]
\end{aligned}
$$

Here we recall that $2 \tau_{\mathrm{E}}^{\mathrm{e}}=\tau_{\mathrm{E}}^{\mathrm{cl}}+\tau_{\mathrm{E}}^{\mathrm{op}}$. At zero Ehrenfest time, $\tau_{\mathrm{E}}^{\mathrm{e}}=0$, we see as in Ref. 58 that while the initial time dependence is determined by $\tau_{\mathrm{D}}$ (first term of rhs of Eq. (43)), for times larger than $t^{*}$ the $t^{2}$ term in the log will be important. We therefore find a deviation from the classical exponential behavior.

This conclusion still holds at finite Ehrenfest time, up to the inclusion of a time shift $2 \tau_{\mathrm{E}}^{\mathrm{e}}$ as predicted in the recent semiclassical derivation ${ }^{66}$ of the survival probability. The treatment of the screened case is more demanding due to the presence of the RC time $\tau$. However since the pole linked to the dwell time $\tau_{\mathrm{D}}$ is only simple, it is clear that even at incomplete screening, there is no term proportional to $t^{2}$. This is in accordance with the absence of deviations for the interacting admittance. However, the Ehrenfest time dependence will be equivalent to the unscreened one, leading to a time shift. Only for complete screening $(\tau=0)$ it is possible to obtain a simple result, which reads on a log scale

$$
\ln \left[\frac{N \tau_{\mathrm{D}}}{N_{\alpha} N_{\beta}} \delta g_{\alpha \beta}^{\tau=0}(t)\right]=-\frac{t-\tau_{\mathrm{E}}^{\mathrm{op}}}{\tau_{\mathrm{D}}}+\ln \left[\frac{1}{N}-\frac{\delta_{\alpha \beta}}{N_{\alpha}}\right] .
$$

\section{MULTITERMINAL SYSTEM WITH TUNNEL BARRIER}

The calculation of the admittance with tunnel barriers follows the trajectory-based method recently developed by Whitney ${ }^{46}$ for the dc case. We recall here the three main changes in the theory with respect to the transparent case. For more details on the inclusion of tunnel barriers we refer to Ref. 46.

At first, in the presence of tunnel barriers the complex amplitude $A_{\gamma}$ in Eq. (17) is extended to include the tunneling probabilities reading 46

$$
A_{\gamma}=C_{\gamma}^{1 / 2} t_{\beta, i} t_{\alpha, j} \prod_{\beta^{\prime}, j^{\prime}}\left[r_{\beta^{\prime}, j^{\prime}}\right]^{\mathcal{N}_{\gamma}\left(\beta^{\prime}, j^{\prime}\right)},
$$

where $C_{\gamma}=\left|\left(\mathrm{d} p_{x_{0}} / \mathrm{d} x\right)_{\gamma}\right|$ is the rate of change in the initial momentum $p_{x_{0}}$ for the exit position $x$ of $\gamma, \mathcal{N}_{\gamma}\left(\beta^{\prime}, j^{\prime}\right)$ is the number of times that $\gamma$ is reflected back into the system from the tunnel barrier on lead $\beta^{\prime}$ and the transmission and refection amplitudes at the lead $\beta$ satisfy $\left|t_{\beta, i}\right|^{2}=\left(1-\left|r_{\beta, i}\right|^{2}\right)=\Gamma_{\beta, i}$. We note that without any loss of generality, we associated in Eq. (45) the momentum $p_{x_{0}}$ (or $p_{x}$ ) with the channel $i$ (or $j$ ).

At this point the replacement of the semiclassical amplitudes by their corresponding classical probabilities still holds, though the tunneling probabilities are included. As an example the probability to go from a phase point $\mathbf{X}_{0}$ (here we associate the channel $i$ to the momentum $p_{\mathrm{F}} \cos \theta_{0}$ ) on lead $\beta$ to an arbitrary point on lead $\alpha$ simply satisfies (for $\alpha \neq \beta$ )

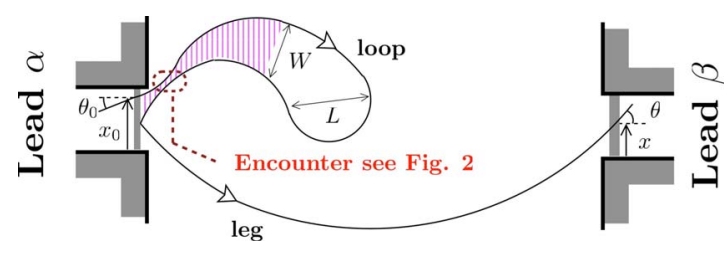

FIG. 3. (Color online) A failed coherent-backscattering contribution to ac conductance, $g_{\alpha \beta}^{u \text {,cbs }}(\omega)$. It involves paths which return close but antiparallel to themselves at lead $\alpha$ but are reflected off the tunnel barrier remaining in the cavity to finally escape via lead $\beta$. The cross-hatched area denotes the region where the two solid paths are paired (within $W_{\alpha} \simeq W$ of each other).

$$
\int_{0}^{\infty} \mathrm{d} t \int_{\alpha} \mathrm{d} \mathbf{X}\left\langle P\left(\mathbf{X}, \mathbf{X}_{0} ; t\right)\right\rangle=\frac{\Gamma_{\beta, i} \Gamma_{\alpha}^{(1)}}{\mathcal{N}},
$$

where we let $\Gamma_{\beta}^{(1)}=\Sigma_{j=1}^{N_{\beta}} \Gamma_{\beta, j}$ and define $\mathcal{N}=\Sigma_{\alpha} \Gamma_{\alpha}^{(1)}$.

More importantly, the introduction of a tunnel barrier induces three changes: (i) the dwell time (single-path survival time) becomes

$$
\tau_{\mathrm{D} 1}^{-1}=\tau_{\mathrm{H}}^{-1} \sum_{\alpha} \Gamma_{\alpha}^{(1)}=\tau_{\mathrm{H}}^{-1} \mathcal{N}
$$

because a typical path may hit a lead but be reflected off the tunnel barrier (remaining in the cavity) numerous times before tunneling and escaping.

(ii) The paired-paths survival time for paths closer than the lead width is no longer equal to the dwell time instead it is given by

$$
\tau_{\mathrm{D} 2}^{-1}=\tau_{\mathrm{H}}^{-1} \sum_{\alpha}\left(2 \Gamma_{\alpha}^{(1)}-\Gamma_{\alpha}^{(2)}\right)=\tau_{\mathrm{H}}^{-1}(2 \mathcal{N}-\tilde{\mathcal{N}}),
$$

where $\Gamma_{\alpha}^{(2)}=\sum_{i=1}^{N_{\alpha}} \Gamma_{\alpha, i}^{2}$ and we define $\tilde{\mathcal{N}}=\Sigma_{\alpha} \Gamma_{\alpha}^{(2)}$. This is because a second path following a path which has not escaped will hit the same tunnel barrier and thus may escape even though the first path did not. Compare this with a system without tunnel barriers: there a path has not escaped because it has not touched the leads; thus a second path following the first one has no possibility to escape.

(iii) The coherent-backscattering peak contributes to transmission as well as reflection. The positive contribution to the transmission competes with the usual negative weaklocalization contribution to transmission, see also Fig. 3.

For the calculation of the Drude conductance, only change (i) above is required, yielding

$$
g_{\alpha \beta}^{u, \mathrm{D}}(\omega)=\Gamma_{\beta}^{(1)} \delta_{\alpha \beta}-\frac{\Gamma_{\alpha}^{(1)} \Gamma_{\beta}^{(1)}}{\mathcal{N}} \frac{1}{1-i \omega \tau_{\mathrm{D} 1}} .
$$

When calculating the conventional weak-localization contribution we need changes (i) and (ii) above. Since the classical paths considered stay close to itself for a time $T_{W}(\epsilon) / 2$ on either side of the encounter we must use the paired-paths survival time, $\tau_{\mathrm{D} 2}$, for these parts of the path. Elsewhere the escape time is given by the single-path survival time, $\tau_{\mathrm{D} 1}$. With these new ingredients we find that the conventional weak-localization contribution becomes 


$$
g_{\alpha \beta}^{u, \mathrm{wl}}(\omega)=\frac{\Gamma_{\alpha}^{(1)} \Gamma_{\beta}^{(1)}}{\mathcal{N}^{2}} \frac{\left(2-\frac{\tilde{\mathcal{N}}}{\mathcal{N}}\right)-2 i \omega \tau_{\mathrm{D} 1}}{\left(1-i \omega \tau_{\mathrm{D} 1}\right)^{3}} e^{-\Theta_{\tau_{\mathrm{E}}} e^{i \omega\left(\tau_{\mathrm{E}} \mathrm{c}+\tau_{\mathrm{E}}^{\mathrm{op}}\right)}}
$$

with $\Theta_{\tau_{\mathrm{E}}}=\tau_{\mathrm{E}}^{\mathrm{op}} / \tau_{\mathrm{D} 2}+\left(\tau_{\mathrm{E}}^{\mathrm{cl}}-\tau_{\mathrm{E}}^{\mathrm{op}}\right) / \tau_{\mathrm{D} 1}$. The exponential suppression $\exp \left(-\Theta_{\tau_{\mathrm{E}}}\right)$ related to the classical correlation is simply the probability that the path segments survive a time $\tau_{\mathrm{E}}^{\mathrm{op}}$ as a pair $\left(\tau_{\mathrm{E}}^{\mathrm{op}} / 2\right.$ on either side of the crossing) and survive an additional time $\left(\tau_{\mathrm{E}}^{\mathrm{cl}}-\tau_{\mathrm{E}}^{\mathrm{op}}\right)$ unpaired (to complete a loop of length $\left.\tau_{\mathrm{E}}^{\mathrm{cl}}\right)$. Similarly as for the transparent case, the exponential dependence $\exp \left[i \omega\left(\tau_{\mathrm{E}}^{\mathrm{cl}}+\tau_{\mathrm{E}}^{\mathrm{op}}\right)\right]$ indicates that the minimal duration of a weak-localization trajectory is $\tau_{\mathrm{E}}^{\mathrm{cl}}+\tau_{\mathrm{E}}^{\mathrm{op}}$.

However as realized by Whitney, ${ }^{46}$ this is not the total weak-localization contribution to conductance because of failed coherent backscattering $g^{u, \mathrm{cbs}}(\omega)$ that contributes to conductance [change (iii) above]. We recall that this involves a path which returns close but antiparallel to itself at lead $\alpha$ but is then reflected off the tunnel barrier on lead $\alpha$ remaining in the cavity until it eventually escapes through lead $\beta$. An example of such a trajectory is shown in Fig. 3. We can calculate the backscattering contribution as before but using $\tau_{\mathrm{D} 2}$, when the paths are within $W_{\alpha}$ of each other, and $\tau_{\mathrm{D} 1}$ elsewhere. This result is then multiplied by the probability that the path reflects off lead $\alpha$ and then escapes through lead $\beta$ and weighted by the dynamical factor $\left(1-i \omega \tau_{\mathrm{D} 1}\right)^{-1}$ due to the diagonal transmission from $\alpha$ to $\beta$, i.e., the leg part of Fig. 3. In addition to the coherent-backscattering expression for $r^{u, \mathrm{cbs}}(\omega)$ this gives a contribution to the admittance of the form

$$
\begin{aligned}
& g_{\alpha \beta}^{u, \mathrm{cbs} 1}(\omega)=\frac{\Gamma_{\alpha}^{(2)}-\Gamma_{\alpha}^{(1)}}{\left(1-i \omega \tau_{\mathrm{D} 1}\right)^{2}} \frac{\Gamma_{\beta}^{(1)}}{\mathcal{N}^{2}} e^{-\Theta_{\tau_{\mathrm{E}}}} e^{i \omega\left(\tau_{\mathrm{E}}^{\mathrm{cl}}+\tau_{\mathrm{E}}^{\mathrm{op}}\right)}, \\
& g_{\alpha \beta}^{u, \mathrm{cbs} 2}(\omega)=\frac{\Gamma_{\beta}^{(2)}-\Gamma_{\beta}^{(1)}}{\left(1-i \omega \tau_{\mathrm{D} 1}\right)^{2}} \frac{\Gamma_{\alpha}^{(1)}}{\mathcal{N}^{2}} e^{-\Theta_{\tau_{\mathrm{E}}}} e^{i \omega\left(\tau_{\mathrm{E}}^{\mathrm{c}}+\tau_{\mathrm{E}}^{\mathrm{op}}\right)}, \\
& r_{\alpha \beta}^{u, \mathrm{cbs}}(\omega)=-\frac{\delta_{\alpha \beta}}{1-i \omega \tau_{\mathrm{D} 1}} \frac{\Gamma_{\alpha}^{(2)}}{\mathcal{N}} e^{-\Theta_{\tau_{\mathrm{E}}}} e^{i \omega\left(\tau_{\mathrm{E}}^{\mathrm{cl}}+\tau_{\mathrm{E}}^{\mathrm{op}}\right)},
\end{aligned}
$$

where we recall that $\Gamma_{\alpha}^{(2)}=\sum_{i=1}^{N_{\alpha}} \Gamma_{\alpha, i}^{2}$.

Using Eqs. (49)-(51), the unscreened admittance in the presence of tunnel barriers reads

$$
\begin{aligned}
\left\langle g_{\alpha \beta}^{u}(\omega)\right\rangle= & \Gamma_{\alpha}^{(1)} \delta_{\alpha \beta}-\frac{\Gamma_{\alpha}^{(1)} \Gamma_{\beta}^{(1)}}{\mathcal{N}\left(1-i \omega \tau_{\mathrm{D} 1}\right)}+\frac{\Gamma_{\alpha}^{(1)} \Gamma_{\beta}^{(1)}}{\mathcal{N}^{2}} \frac{e^{-\Theta} \tau_{\mathrm{E}} e^{i \omega\left(\tau_{\mathrm{E}}^{\mathrm{cl}}+\tau_{\mathrm{E}}^{\mathrm{op}}\right)}}{\left(1-i \omega \tau_{\mathrm{D} 1}\right)} \\
& \times\left(\frac{2-\tilde{\mathcal{N}} / \mathcal{N}-2 i \omega \tau_{\mathrm{D} 1}}{\left(1-i \omega \tau_{\mathrm{D} 1}\right)^{2}}+\frac{\Gamma_{\alpha}^{(2)} / \Gamma_{\alpha}^{(1)}+\Gamma_{\beta}^{(2)} / \Gamma_{\beta}^{(1)}-2}{\left(1-i \omega \tau_{\mathrm{D} 1}\right)}\right. \\
& \left.-\frac{\Gamma_{\alpha}^{(2)}}{\Gamma_{\alpha}^{(1)}} \frac{\mathcal{N}}{\Gamma_{\beta}^{(1)}} \delta_{\alpha \beta}\right)+\mathcal{O}\left(N^{-1}\right) .
\end{aligned}
$$

As a check of the formula (52), we can easily recover the previous Eq. (38) for the unscreened admittance obtained for transparent barriers and also the tunnel dc conductance. ${ }^{46}$ After the substitution of Eq. (52) into Eq. (15) the screened admittance in presence of tunnel barriers reads

$$
\begin{aligned}
\left\langle g_{\alpha \beta}(\omega)\right\rangle= & \Gamma_{\alpha}^{(1)} \delta_{\alpha \beta}-\frac{\Gamma_{\alpha}^{(1)} \Gamma_{\beta}^{(1)}}{\mathcal{N}(1-i \omega \tau)}+\frac{\Gamma_{\alpha}^{(1)} \Gamma_{\beta}^{(1)}}{\mathcal{N}^{2}} \frac{e^{-\Theta} \tau_{\mathrm{E}} e^{i \omega\left(\tau_{\mathrm{E}}^{\mathrm{cl}}+\tau_{\mathrm{E}}^{\mathrm{op}}\right)}}{\left(1-i \omega \tau_{\mathrm{D} 1}\right)} \\
& \times\left(\frac{2-\tilde{\mathcal{N}} / \mathcal{N}-2 i \omega \tau}{(1-i \omega \tau)^{2}}+\frac{\Gamma_{\alpha}^{(2)} / \Gamma_{\alpha}^{(1)}+\Gamma_{\beta}^{(2)} / \Gamma_{\beta}^{(1)}-2}{(1-i \omega \tau)}\right. \\
& \left.-\frac{\Gamma_{\alpha}^{(2)}}{\Gamma_{\alpha}^{(1)}} \frac{\mathcal{N}}{\Gamma_{\beta}^{(1)}} \delta_{\alpha \beta}\right)+\mathcal{O}\left(N^{-1}\right),
\end{aligned}
$$

where the quantum RC time reads now $\tau^{-1}=\tau_{\mathrm{D} 1}^{-1}+\mathcal{N} G_{0} / C$. We emphasize that from Eq. (53) it is possible to derive all the results presented in this paper and therefore this equation is the central result of this paper.

In the second line of Eq. (53), the second contribution in the brackets represents the correction due to the presence of the failed coherent backscattering. Importantly, Eq. (53) includes both, the limit of infinite capacitance $C$ and the transparent case. In the charge neutrality limit $(\tau=0)$ the presence of the tunnel barriers does not drastically alter the conclusion drawn for the transparent case. Indeed, for the weaklocalization correction, in addition to the expected substitution $N_{\alpha}, N$ by $\Gamma_{\alpha}^{(1)}, \mathcal{N}$, we observe only a renormalization by a factor $\left(\Gamma_{\alpha}^{(2)} / \Gamma_{\alpha}^{(1)}+\Gamma_{\beta}^{(2)} / \Gamma_{\beta}^{(1)}-\tilde{\mathcal{N}} / \mathcal{N}\right)$. Thus Eq. (41) becomes

$$
g_{\alpha \beta}^{\mathrm{wl}, \tau=0}(\omega)=\left(\frac{\Gamma_{\alpha}^{(2)}}{\Gamma_{\alpha}^{(1)}}+\frac{\Gamma_{\beta}^{(2)}}{\Gamma_{\beta}^{(1)}}-\frac{\tilde{\mathcal{N}}}{\mathcal{N}}\right) \frac{\Gamma_{\alpha}^{(1)} \Gamma_{\beta}^{(1)}}{\mathcal{N}^{2}} \frac{e^{-\Theta} \tau_{\tau_{\mathrm{E}}} e^{i \omega\left(\tau_{\mathrm{E}}^{\mathrm{cl}}+\tau_{\mathrm{E}}^{\mathrm{op}}\right)}}{\left(1-i \omega \tau_{\mathrm{D} 1}\right)} .
$$

More importantly, one of the main effects of the tunnel barrier in the dc case was the suppression of the weaklocalization correction ${ }^{46,73}$ for opaque barriers. This suppression results from the competition between two purely quantum effects, interference and tunneling. The corresponding semiclassical treatment ${ }^{46}$ shows that the cancellation is due to an exact compensation between the weak-localization correction and the failed coherent backscattering. It is interesting that this conclusion cannot be generalized to ac transport. Since the frequency dependence of the weaklocalization correction differs from the one of the failed coherent backscattering the compensation cannot occur. Dynamical weak localization is thus more robust against the presence of tunnel barriers. We note, however, that for $\tau=0$ we recover the cancellation of the weak-localization correction with tunnel probabilities, see Eq. (54).

\section{CHARGE-RELAXATION RESISTANCE OF A MESOSCOPIC CHAOTIC CAPACITOR}

To illustrate and apply the general results derived above, we consider here the mesoscopic equivalent of a classical RC circuit. ${ }^{23}$ A quantum coherent capacitor has been recently investigated experimentally by Gabelli et al. ${ }^{8}$ using a twodimensional electron gas. The quantum capacitor is composed of a macroscopic metallic electrode on top of a lateral quantum dot defining the second electrode. The role of the resistance is played by a quantum-point contact that connects 


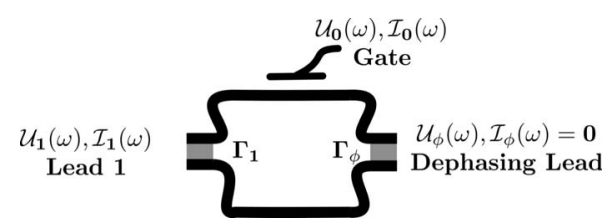

FIG. 4. Schematic picture of the mesoscopic capacitor with the dephasing lead (see text). The chaotic cavity has an extra lead (lead $\phi)$, whose voltage is chosen to render the net current zero, which leads to dephasing without a loss of particles. Since each channel has the same tunnel rate $\Gamma_{1, i}=\Gamma$ and $\Gamma_{\phi, i}=\Gamma_{\phi}, \forall i$, the dwell time of the capacitor is $\tau_{\mathrm{D}} \propto(N \Gamma)^{-1}$ and the dephasing time reads $\tau_{\phi}$ $\propto\left(N_{\phi} \Gamma_{\phi}\right)^{-1}$.

the quantum dot to a reservoir. The experiment was performed in the coherent regime at high magnetic field in the one-edge-state limit. Measuring the real and imaginary part of the admittance of such a circuit, Ref. 8 confirmed the predicted $^{23}$ universal value of the quantized chargerelaxation resistance of a single-channel cavity, which is equal to half a resistance quantum $h / 2 e^{2}$.

Based on this experimental realization we propose here to investigate the opposite regime of large channel numbers at zero magnetic field. This regime is not characterized by the universal value of the preceding fully quantum one, however it should be experimentally accessible. If we assume that the quantum dot is chaotic we can map this system to the oneterminal geometry of the more general set up considered in the previous section. The transparency of the quantum-point contact is replaced by the transmission probability of the tunnel barrier $\boldsymbol{\Gamma}_{1}$. To simplify the result we assume in the following that the $N$ channels of the capacitor have the same tunnel rate, i.e., $\Gamma_{1, i}=\Gamma(\forall i)$, the dwell time of the capacitor is thus $\tau_{\mathrm{D}}=\tau_{\mathrm{H}} /(N \Gamma)$.

In a quantum coherent capacitor, there is obviously no dc current but we can address ac transport via the admittance $G(\omega) .{ }^{24,54}$ At low temperatures it is characterized by an electrochemical capacitance $C_{\mu}$ and a charge-relaxation resistance $R_{q}$

$$
G(\omega)=-i \omega C_{\mu}+\omega^{2} C_{\mu}^{2} R_{q}+\mathcal{O}\left(\omega^{3}\right) .
$$

In contrast to their classical counterparts, $C_{\mu}$ and $R_{q}$ strongly depend on the local density inside the sample. ${ }^{74}$ They are thus sensitive to the phase-coherent dynamics of the electrons inside the sample and thus subject to dephasing.

To model the loss of coherence of electrons inside the cavity we appeal to the so-called voltage/dephasing probe model, ${ }^{75}$ which consists of adding another lead $\phi$, (see Fig. 4) to our cavity and tuning the potential of this probe in such a way that the net current is zero. Consequently any electron that enters this lead is immediately replaced by another one with an unrelated phase leading to incoherence without loss of particles. While such an approach has recently been used for the mesoscopic capacitor in the one channel limit, ${ }^{15}$ here we investigate similar effects of the dephasing in the limit of large channel numbers ${ }^{76}$ where our semiclassical method is fully justified.

The admittance can be written as

$$
G(\omega)=\frac{-i \omega C \chi(\omega)}{-i \omega C+\chi(\omega)},
$$

where

$$
\chi(\omega)=G_{0}\left(g_{11}^{u}(\omega)-\frac{g_{1 \phi}^{u}(\omega) g_{\phi 1}^{u}(\omega)}{g_{\phi \phi}^{u}(\omega)}\right) .
$$

The unscreened admittance elements are given in Eq. (52). The survival times $\tau_{\mathrm{D} 1}$ and $\tau_{\mathrm{D} 2}$ of this two-lead geometry are related to the real dwell time $\tau_{\mathrm{D}}$ of our capacitor and to the dephasing time $\tau_{\phi}=\tau_{\mathrm{H}} /\left(N_{\phi} \Gamma_{\phi}\right)$, where $N_{\phi}$ and $\Gamma_{\phi, i}$ $=\Gamma_{\phi}(\forall i)$ are, respectively, the number of channels and the tunneling rates of the dephasing lead ${ }^{47}$

$$
\begin{gathered}
\tau_{\mathrm{D} 1}=\tau_{\mathrm{D}}\left[1+\frac{\tau_{\mathrm{D}}}{\tau_{\phi}}\right]^{-1}, \\
\tau_{\mathrm{D} 2}=\tau_{\mathrm{D}}\left[(2-\Gamma)+\frac{\tau_{\mathrm{D}}}{\tau_{\phi}}\left(2-\Gamma_{\phi}\right)\right]^{-1} .
\end{gathered}
$$

Inserting expression (52) for the unscreened admittance elements into Eqs. (56) and (57) and performing an expansion in $\omega$ we get

$$
\begin{gathered}
C_{\mu}=\frac{C e^{2} \nu}{C+e^{2} \nu}, \\
G_{0} R_{q}=\frac{1}{\Gamma N}+\frac{D\left(\tau_{\mathrm{E}}^{\mathrm{cl}}, \tau_{\mathrm{E}}^{\mathrm{op}}, \tau_{\phi}\right)}{(\Gamma N)^{2}}+\mathcal{O}\left(N^{-3}\right),
\end{gathered}
$$

where we additionally used the relation between the mean density of states, $\nu$, and the dwell time, $\tau_{\mathrm{D}}=h \nu /\left(d_{s} N \Gamma\right)$. The dephasing function $D\left(\tau_{\mathrm{E}}^{\mathrm{cl}}, \tau_{\mathrm{E}}^{\mathrm{op}}, \tau_{\phi}\right)$ reads

$$
D\left(\tau_{\mathrm{E}}^{\mathrm{cl}}, \tau_{\mathrm{E}}^{\mathrm{op}}, \tau_{\phi}\right)=\Gamma e^{-\tau_{\mathrm{E}}^{\mathrm{op}} / \tau_{\mathrm{D}}(1-\Gamma)-\tau_{\mathrm{E}}^{\mathrm{cl}} / \tau_{\mathrm{D}}} \frac{e^{-\tau_{\mathrm{E}}^{\mathrm{op}} / \tau_{\phi}\left(1-\Gamma_{\phi}\right)-\tau_{\mathrm{E}}^{\mathrm{cl}} / \tau_{\phi}}}{\left(1+\frac{\tau_{\mathrm{D}}}{\tau_{\phi}}\right)} .
$$

We finally consider the effect of a magnetic flux on the charge-relaxation resistance. Substituting Eq. (39) (the dwell time being replaced by the survival time $\tau_{\mathrm{D} 1}$ ) into Eq. (56) leaves the electrochemical capacitance $C_{\mu}$ unchanged; only the dephasing function $D\left(\tau_{\mathrm{E}}^{\mathrm{cl}}, \tau_{\mathrm{E}}^{\mathrm{op}}, \tau_{\phi}\right)$ is affected and replaced by

$$
D\left(\tau_{\mathrm{E}}^{\mathrm{cl}}, \tau_{\mathrm{E}}^{\mathrm{op}}, \tau_{\phi}, \Phi\right)=\Gamma e^{-\tau_{\mathrm{E}}^{\mathrm{op}}(1-\Gamma) / \tau_{\mathrm{D}}-\tau_{\mathrm{E}}^{\mathrm{cl}} / \tau_{\mathrm{D}}} \frac{e^{-\tau_{\mathrm{E}}^{\mathrm{op}}\left(1-\Gamma_{\phi}\right) / \tau_{\phi}-\tau_{\mathrm{E}}^{\mathrm{cl}} / \tau_{\phi}}}{\left(1+\mathcal{A}^{2} \Phi^{2} \frac{\tau_{\mathrm{D}}}{\tau_{\mathrm{f}}}+\frac{\tau_{\mathrm{D}}}{\tau_{\phi}}\right)} .
$$

From this semiclassical investigation of the charge-relaxation resistance, we can see that the fully coherent limit $\left(\tau_{\phi}=\infty\right.$, $\tau_{\mathrm{E}}^{\mathrm{op}}=\tau_{\mathrm{E}}^{\mathrm{cl}}=0$, and $\Phi=0$ ) delivers

$$
R_{q}=\frac{1}{G_{0}} \frac{1}{\Gamma N}\left(1+\frac{1}{N}\right)+\mathcal{O}\left(N^{-3}\right) .
$$

Equation (62) is the first derivation of the chargerelaxation resistance in the large $N$ limit in presence of tun- 
nel barriers. While the leading order was guessed, ${ }^{55}$ the weak-localization correction to $R_{q}$ has never been calculated before. Surprisingly, it is linear in the inverse tunnel rate $\Gamma^{-1}$, indicating that the calculation of the subleading-order correction cannot be simply obtained by an effective renormalization of the channel number $N_{\text {eff }}=\Gamma N$.

For the incoherent limit, obtained either by $\tau_{\mathrm{E}} \rightarrow \infty, \Phi$ $\rightarrow \infty$, or $\tau_{\phi}=0$, we get a suppression of the weak-localization correction and thus $R_{q}$ reduces to

$$
R_{q}=\frac{1}{G_{0}} \frac{1}{\Gamma N} .
$$

This value corresponds to the fully incoherent limit that corresponds to the two-terminal resistance and has been obtained under the simple application of our dephasing process. Although the edge-state calculation ${ }^{15}$ considers a different limit $(N=1)$ than our semiclassical calculation and therefore any comparison should be taken with caution, it is interesting to note that the incoherent limit was not trivially obtained in the edge-state calculation where perfect interchannel relaxation inside the voltage probe was assumed. This seems not to be required in the fully chaotic case in the limit $N \gg 1$.

\section{CONCLUSIONS}

In this work we focused on the topic of ac transport through chaotic ballistic conductors, addressing in particular weak-localization corrections to the admittance from a semiclassical perspective. Employing trajectory-based methods we confirmed RMT results for the bare and screened admittance and, going beyond RMT, derived the Ehrenfest time dependence. The Ehrenfest time scale enters twice into the expressions for dynamical weak localization: first, as an exponential suppression with an exponent given by the ratio of the Ehrenfest and dwell time, $\tau_{\mathrm{E}} / \tau_{\mathrm{D}}$; second the dynamical weak localization acquires an oscillatory frequencydependent behavior of period $2 \tau_{\mathrm{E}}$, which may be amenable to measurements based on variations in the ac frequency. We emphasize that our results are valid for any finite capacitance
$C$ and hence not limited to the electroneutrality assumption of Ref. 34. This extends the class of experimental settings for which the Ehrenfest time correction can be investigated. More generally, the results presented underline, first, the power of semiclassical techniques to provide a clear and quantitative picture of ac-driven quantum transport in the various regimes and, second, they give a justification of the stub model ${ }^{26,33}$ in the low-frequency regime.

Moreover we took into account tunnel barriers in the semiclassical approach to the ac admittance, extending the work of Whitney ${ }^{46}$ on dc transport. This led us to a general formulation of ac transport. One main conclusion is that weak-localization is more robust against effects of tunnel barriers in the dynamical than in the dc regime. The extension of our semiclassical treatment to tunnel barriers also enables us to access the experimentally relevant case of a quantum-coherent capacitor, for which we provide the first derivation of the weak-localization correction to the charge relaxation resistance in presence of tunnel barriers.

We add that the semiclassical approach presented has recently been extended toward a semiclassical understanding of the proximity effect on the density of states of chaotic Andreev billiards. ${ }^{77}$ Finally, the ac conductance discussed here is closely related to problems of computing (photo)absorption and, more generally, linear-response-based dynamical susceptibilities for mesoscopic quantum systems. It appears promising to apply the semiclassical techniques, developed here for (ac-)quantum transport, to refine earlier semiclassical approaches ${ }^{52}$ to (photo)absorption in closed ballistic cavities or metal clusters, which additionally poses the challenge to semiclassically cope with screening effects and plasmon excitations.

\section{ACKNOWLEDGMENTS}

The authors thank P. W. Brouwer, M. Büttiker, M. Gutiérrez, S. Nigg, M. Polianski, and R. S. Whitney for valuable and stimulating discussions. We acknowledge funding from the DFG under GRK 638, FOR 760 and from the Alexander von Humboldt Foundation (C.P. and J.K.).
${ }^{1}$ J. B. Pieper and J. C. Price, Phys. Rev. Lett. 72, 3586 (1994).

${ }^{2}$ W. Chen, T. P. Smith, M. Büttiker, and M. Shayegan, Phys. Rev. Lett. 73, 146 (1994).

${ }^{3}$ L. P. Kouwenhoven, S. Jauhar, J. Orenstein, P. L. McEuen, Y. Nagamune, J. Motohisa, and H. Sakaki, Phys. Rev. Lett. 73, 3443 (1994).

${ }^{4}$ M. Reznikov, M. Heiblum, H. Shtrikman, and D. Mahalu, Phys. Rev. Lett. 75, 3340 (1995).

${ }^{5}$ S. Verghese, R. A. Wyss, T. Schapers, Q. Hu, A. Förster, and M. J. Rooks, Phys. Rev. B 52, 14834 (1995).

${ }^{6}$ R. J. Schoelkopf, P. J. Burke, A. A. Kozhevnikov, D. E. Prober, and M. J. Rooks, Phys. Rev. Lett. 78, 3370 (1997).

${ }^{7}$ L. H. Reydellet, P. Roche, D. C. Glattli, B. Etienne, and Y. Jin, Phys. Rev. Lett. 90, 176803 (2003).

${ }^{8}$ J. Gabelli, G. Fève, J. Berroir, and B. Placais, Science 313, 499
(2006).

${ }^{9}$ G. Fève, A. Mahé, J.-M. Berroir, T. Kontos, B. Plaçais, D. Glattli, A. Cavanna, B. Etienne, and Y. Jin, Science 316, 1169 (2007).

${ }^{10}$ K. E. Nagaev, S. Pilgram, and M. Büttiker, Phys. Rev. Lett. 92, 176804 (2004).

${ }^{11}$ F. W. J. Hekking and J. P. Pekola, Phys. Rev. Lett. 96, 056603 (2006).

${ }^{12}$ J. Salo, F. W. J. Hekking, and J. P. Pekola, Phys. Rev. B 74, 125427 (2006).

${ }^{13}$ M. L. Polianski, P. Samuelsson, and M. Büttiker, Phys. Rev. B 72, 161302(R) (2005).

${ }^{14}$ D. Bagrets and F. Pistolesi, Phys. Rev. B 75, 165315 (2007).

${ }^{15}$ S. E. Nigg and M. Büttiker, Phys. Rev. B 77, 085312 (2008).

${ }^{16}$ M. Moskalets, P. Samuelsson, and M. Büttiker, Phys. Rev. Lett. 
100, 086601 (2008).

${ }^{17}$ I. Safi, C. Bena, and A. Crépieux, Phys. Rev. B 78, 205422 (2008).

${ }^{18}$ H. C. Park and K.-H. Ahn, Phys. Rev. Lett. 101, 116804 (2008).

${ }^{19}$ D. S. Fisher and P. A. Lee, Phys. Rev. B 23, 6851 (1981).

${ }^{20}$ H. U. Baranger and A. D. Stone, Phys. Rev. B 40, 8169 (1989).

${ }^{21}$ K. Shepard, Phys. Rev. B 43, 11623 (1991).

${ }^{22}$ H. M. Pastawski, Phys. Rev. B 44, 6329 (1991).

${ }^{23}$ M. Büttiker, A. Prêtre, and H. Thomas, Phys. Rev. Lett. 70, 4114 (1993).

${ }^{24}$ M. Büttiker, J. Phys.: Condens. Matter 5, 9361 (1993).

${ }^{25}$ M. G. Vavilov, J. Phys. A 38, 10587 (2005).

${ }^{26}$ M. Polianski and P. W. Brouwer, J. Phys. A 36, 3215 (2003).

${ }^{27}$ M. Büttiker and T. Christen, in Mesoscopic Electron Transport, NATO Advanced Studies Institute, Series E: Applied Science, edited by L. Kowenhoven, G. Schön, and L. Sohn (Kluwer, Dordrecht, 1997), p. 259

${ }^{28}$ The regime of larger ac-driving frequency requires other techniques, such as Floquet scattering theory which has been applied in the context of ac-driven chaotic scattering, e.g. in Ref. 78.

${ }^{29}$ R. Blümel and U. Smilansky, Phys. Rev. Lett. 60, 477 (1988).

${ }^{30}$ H. U. Baranger, R. A. Jalabert, and A. D. Stone, Phys. Rev. Lett. 70, 3876 (1993).

${ }^{31}$ J. Verbaarschot, H. Weidenmüller, and M. Zirnbauer, Phys. Rep. 129, 367 (1985).

${ }^{32}$ K. Frahm, EPL 30, 457 (1995).

${ }^{33}$ P. W. Brouwer and M. Büttiker, EPL 37, 441 (1997).

${ }^{34}$ I. L. Aleiner and A. I. Larkin, Phys. Rev. B 54, 14423 (1996).

${ }^{35}$ A. I. Larkin and Yu. N. Ovchinnikov, Zh. Eksp. Teor. Fiz. 55, 2262 (1968) [Sov. Phys. JETP 28, 1200 (1969)].

${ }^{36}$ G. Berman and G. Zaslavsky, Physica A 91, 450 (1978).

${ }^{37}$ M. G. Vavilov and A. I. Larkin, Phys. Rev. B 67, 115335 (2003).

${ }^{38}$ H. Schomerus and P. Jacquod, J. Phys. A 38, 10663 (2005).

${ }^{39}$ D. Waltner and K. Richter, in Handbook of Nonlinear Dynamics in Nanosystems, edited by G. Radons et al. (Wiley, New York, in press).

${ }^{40}$ K. Richter and M. Sieber, Phys. Rev. Lett. 89, 206801 (2002).

${ }^{41}$ I. Adagideli, Phys. Rev. B 68, 233308 (2003).

${ }^{42}$ S. Müller, S. Heusler, P. Braun, and F. Haake, New J. Phys. 9, 12 (2007).

${ }^{43}$ P. Jacquod and R. S. Whitney, Phys. Rev. B 73, 195115 (2006).

${ }^{44}$ P. W. Brouwer and S. Rahav, Phys. Rev. B 74, 075322 (2006).

${ }^{45}$ P. W. Brouwer, Phys. Rev. B 76, 165313 (2007).

${ }^{46}$ R. S. Whitney, Phys. Rev. B 75, 235404 (2007).

${ }^{47}$ C. Petitjean, P. Jacquod, and R. S. Whitney, Pis'ma Zh. Eksp. Teor. Fiz. 86, 736 (2007) [JETP Lett. 86, 647 (2007)].

${ }^{48}$ R. S. Whitney, P. Jacquod, and C. Petitjean, Phys. Rev. B 77, 045315 (2008).

${ }^{49}$ J. Kuipers and M. Sieber, Phys. Rev. E 77, 046219 (2008).

${ }^{50}$ P. W. Brouwer and A. Altland, Phys. Rev. B 78, 075304 (2008).

${ }^{51}$ M. Wilkinson, J. Phys. A 20, 2415 (1987).

${ }^{52}$ B. Mehlig and K. Richter, Phys. Rev. Lett. 80, 1936 (1998).
${ }^{53}$ K. Richter, Semiclassical Theory of Mesoscopic Quantum Systems, Springer Tracts in Modern Physics (Springer, Berlin, 2000), Vol. 161.

${ }^{54}$ V. A. Gopar, P. A. Mello, and M. Büttiker, Phys. Rev. Lett. 77, 3005 (1996).

${ }^{55}$ Y. Blanter and M. Büttiker, Phys. Rep. 336, 1 (2000).

${ }^{56}$ A. Prêtre, H. Thomas, and M. Büttiker, Phys. Rev. B 54, 8130 (1996).

${ }^{57}$ J. Wang, Q. Zheng, and H. Guo, Phys. Rev. B 55, 9770 (1997).

${ }^{58}$ M. Büttiker, J. Low Temp. Phys. 118, 519 (2000).

${ }^{59}$ I. Aleiner, P. Brouwer, and L. Glazman, Phys. Rep. 358, 309 (2002).

${ }^{60}$ P. W. Brouwer, A. Lamacraft, and K. Flensberg, Phys. Rev. B 72, 075316 (2005).

${ }^{61}$ B. Wang, J. Wang, and H. Guo, Phys. Rev. Lett. 82, 398 (1999).

${ }^{62}$ W. H. Miller, Adv. Chem. Phys. 30, 77 (1975).

${ }^{63}$ M. C. Gutzwiller, Chaos in Classical and Quantum Mechanics (Springer, New York, 1990).

${ }^{64}$ M. Sieber, J. Phys. A 32, 7679 (1999).

${ }^{65}$ Part IV "time dependence" of Ref. 44 . In order to compare with our result, one should at first neglect the difference between $\tau_{\mathrm{E}}^{\mathrm{cl}}$ and $\tau_{\mathrm{E}}^{\mathrm{op}}$, then you substitute $\tau_{\mathrm{abs}}$ by $-i \omega$ in Eqs. (52) and (53) of Ref. 44.

${ }^{66}$ D. Waltner, M. Gutiérrez, A. Goussev, and K. Richter, Phys. Rev. Lett. 101, 174101 (2008).

${ }^{67}$ M. Gutiérrez, D. Waltner, J. Kuipers, and K. Richter, Phys. Rev. E 79, 046212 (2009).

${ }^{68}$ As previously fixed in the dc case see Refs. 44 and 43. Reference 34 had incorrectly treated the classical correlation at the encounter and therefore presented a discrepancy of a factor 2 concerning the ratio $\tau_{\mathrm{E}}^{\mathrm{cl}} / \tau_{\mathrm{D}}$.

${ }^{69}$ S. Oberholzer, E. V. Sukhorukov, and C. Schönenberger, Nature (London) 415, 765 (2002).

${ }^{70}$ O. Yevtushenko, G. Lütjering, D. Weiss, and K. Richter, Phys. Rev. Lett. 84, 542 (2000).

${ }^{71}$ G. Casati, G. Maspero, and D. L. Shepelyansky, Phys. Rev. E 56, R6233 (1997)

${ }^{72}$ K. M. Frahm, Phys. Rev. E 56, R6237 (1997).

${ }^{73}$ S. Iida, H. A. Weidenmüller, and J. A. Zuk, Ann. Phys. 200, 219 (1990).

${ }^{74}$ M. Büttiker, J. Korean Phys. Soc. 34, 121 (1999).

${ }^{75}$ M. Büttiker, Phys. Rev. B 33, 3020 (1986).

${ }^{76}$ Strictly speaking, dephasing, and voltage probe models are fully equivalent in the one channel limit only. However, they also lead to the same results in the limit of low temperature and frequency. See H. Förster, P. Samuelsson, S. Pilgram, and M. Büttiker, Phys. Rev. B 75, 035340 (2007).

${ }^{77}$ J. Kuipers, C. Petitjean, D. Waltner, and K. Richter, arXiv:0907.2660 (unpublished).

${ }^{78}$ M. Henseler, T. Dittrich, and K. Richter, Phys. Rev. E 64, 046218 (2001). 